\title{
Aspectos vivenciales en niños sobrevivientes de cáncer infantil
}

\author{
Carlos Almonte V.'; Pilar Cubillos P. 2; Eugenia Emparanza del S. ${ }^{3}$
}

\section{Emotional aspects in childhood cancer survivors}

\begin{abstract}
Emotiona recctons among crildren loge 11 year or lessł sunviving carcer were investigated. Most of them perceived world in o threarering way, selt serceptions were predcminantiy negative ano their moin cause of cnxety was death. These findings highilghi tine need to give due consideration to the emolional aspects of these palients olong diagnostic procedires, ireatment and further controls. Children with carcer cnd their families should be offered sound emotional support and appropriate informction aboul the froblem and is manogement to improve their quolity of life.
\end{abstract}

(Key words cancer, neoplosm, chid, survival, afective symptons, enrotions !

La sobrevida de los niños afectados por las diversas formas de cáncer infantil ha aumentado significativamente en los últimos 20 años, 10 que puede atribuirse a los avances en los métodos de diagnóstico, estrategias de manejo y esquemas terapéuticos ${ }^{1-5}$. Estos resultados plantean a los especialistas la necesidad de atender el compromiso en las distintas áreas del desarrollo físico y psíquico (incluyendo la dimensión afectiva y cognitiva) de los afectados. El conocimiento del impacto de la enfermedad en la persona del niño permite un tratamiento más integral, favoreciendo un ajuste psicosocial, actual y futuro, satisfactorio y mejor calidad de vida, la que en último término se traduce en vivencias de bienestar personal ${ }^{12}$. El niño con cáncer debe lidiar con una serie de agresiones a lo largo de su enfermedad: técnicas de diagnóstico complejas, invasoras y a veces dolorosas; trataniento generalmente agresivo; alejamiento transitorio del hogar por hospitalización en una etapa de gran dependencia de los adultos; repercusiones emocionales y económicas en la fami-

1. Servicio de Salud Mental, Hospital Roberio del Río. Universidad de Chile.

2. Psicóloga.

3. Unidad de Hematología - Oncología, Hospital Roberto del Río. Departamento de Pediatría, Universidad de Chile. Proyecto 854, Investigación Fondecyt 1992. Estudio calidad de vida de sobrevivientes de cáncer infantil. lia; temores de desenlace fatal. Ellos lo ponen en una situación de gran vulnerabilidad, propicia para perturbaciones del desarrollo psíqui$\mathrm{co}^{4.9}$. Así, pues, es necesario evaluar cómo el niño sobreviviente de cáncer ha sido impactado por su enfermedad, en particular sobre los aspectos vivenciales, que constituyen la dimensión que ha recibido menor atención en las investigaciones sobre esta materia y que, por esta razón, fueron elegidos para este estudio.

El esclarecimiento de las vivencias infantiles permite entender y forjar herramientas para ayudar a los niños afectados y a sus familias, que no comprenden y muchas veces no hablan de sus temores. De este modo es posible prevenir el asentamiento de núcleos de patología psíquica, contribuyendo a mejorar la calidad de la sobrevida.

La enfermedad oncol6gica ocurre en las primeras etapas del desarrollo infantil en la población estudiada, por lo tanto cabría esperar mayor vulnerabilidad a la angustia que en otras edades por los incipientes recursos psicológicos con que cuenta el niño para manejarla. Por otra parte, el ajuste escolar puede verse interferido por las reiteradas inasistencias y experiencias negativas en la relación con sus compañeros (como pueden ser las burlas por la caída del cabello o por los efectos y secuelas de las radiaciones en el sistema nervioso central) ${ }^{4}$. 


\section{Pacientes y Método}

Consideramos sobrevivientes de cáncer infantil a los niôos que, habiendo sulrido la enfermedad, se han mantenido sin recaídos durante 5 afoos o más. La muestra de este análisis estaba constituida por 19 niños de ambos sexos (8 niñas) evaluados entre las edades de 6 y 11 años (12 pacientes de dos años o menos, siete de eillos varones; 5 niños de $>2$ a 5 años, 4 varones; dos niñas de $>5$ a 7 años en el momenco de hacer el diagnostico), alendidos en la unidad de oncología del servicio de pediatría del Hospital Roberto del Río, que forman parte de un universo incluido en una investigación sobre la calidad de vida de sobrevivientes de cáncer infantil. Los correspondientes diagnosticos oncológicos cran leucemia linfoblástica aguda $(n=5)$; leucemia mieloide $(n=1)$; linforna de Hodgkin $(n=2)$; tumor testicular (n = 3); tumor de Wilms $(n=3)$ : neuroblastoma ( $\mathrm{n}=3$ ); linfoma mediastínico leucemizado $(\mathrm{n}=$ 1) y hepatoblasioma $(n=1)$. La edad al diagnóstico varió entre 15 días y 6 àios, concentrándose la mayoria de la muestra en los primeros dos atos de vida (63\%). Nueve de los 19 niños quedaron con algún tipo de daño o secuela física ( $48 \%$ de la muestra), tces con orquidectomía, tres con nefrectomía, uno con asimetría facial. uno con hipoacusia y urro con cicarriz quieloidea abdomithal.

Los niños fueron individualmente sometidos a una evaJuación psicologica de su funcionamiento global, en aspectos cognitivos, afectivos y sociales. Su rendimiento escolar se evaluó en base al promedio de notas del año anterior. considerando cuatro categorias: nuy bueno $(6,0-7,0)$. bueno $(5,0-5,9)$, regular $(4,0-4,9)$ y deficiente (inferior a 4,0 o repitencia). Adernás se les aplicó la escala de inteligencia de Weschler para niños, revisada (WISC-R. normas chilenas), y la prueba gestáltica visomotora de Bender Koppitz, para cvaluar el área cognitiva y de madurez visomotora. La relación con los pares se determinó sobre la base de ta información entregads por los profesores, ha entrevista con los padres y el mismo ničo.

Para el diagnóstico de los aspectos vivenciales se emplearon prucbas psicologicas proyectivas basadas en consignas y en apercepción lemática.

Las técnicas proyectivas basadas en consignas se hicieron mediarte pruebas graficas (dibujo libre, de la figura humana, de la familia y del problema). en las gue el nifio dibuja -a partir de sus propias experiencias- lo que para él adqujerc mayor relevancia afectiva, o bien mediante cuentos donde el niño escribe una historia cuyos personajes son animales, debiendo señalar con cuál o cuáles se identifica. La praeba proyectiva de apercepción temática in †antil de L.S. Bellak (CAT-A) y algunas láminas de su forma suplementaria (CAT-S) es un método clásico empleado en el diagnóstico psicológico infantil. en el que se presentaron al niño 13 láminas con motivos animales, debriendo realizar historias con cada una de ellas. Cada lámina explora distintas situaciones a las cuales el nifo se enfrenta en sus relaciones familiares, la percepción del mundo y su experiencia con la enfermedad.

Paca los tines de esta comunicación, a partis de las pruebas proyectivas, defirimos algunas dimensiones que permiten evaluar cualitativamente las vivencias que tiene el niño de sí y de sta mundo: La percepción del mundo alude a la percepción quz tiene el niño del mundo que to rodea (amenazante, peligroso, amistoso y sin grandes peli- gros que amenacen su integridad). En la percepción de si mismo pueden, a su vez, predoninar los senlimientos positivos, de fortaleza para enfrentar las dificultades; Jos negativos, hacia el polo depresive, con sentimientos de desvalimiento y temar, o bien los agresivos. provocando el temor de otros.

Las ansiedades principales identifican las situaciones que generan mayor temor de muerte. daho físico, abandono, soledad, desaprobación, o ansiedad difusa no circunscrita y evalúan la capacidad del niño para controlar la ansiedad o si ésta lo sobrepasa cuando es demasiado intensa. díficultándole un ajuste positivo.

El conflicto principal se refjere al tipo de ternática que el niño propone, la que paede ser persecutoria. de convivercía, de competencia, de extravio o no planieada. Por tipo de solución entendemos la manera como el sujeto resuelve sus conflictos: puede ser positiva (si la solutión permite la supervivencia de los personajes, con una buena adaptación posterior); negativa (la historia termina con la destrucción de los personajes principales o bien el desarrollo queda bluqueado en una situación de impotencia); abierta (no se plantea desenlace, no se resuelve el conflicro).

El tipo de vínculo es el modo como el personaje principal se relaciona con otros, pudiendo predominar una relación agresiva, inesiable, anorosa y solidaria, o no plantearse vinculación entre los personajes.

\section{Resultados}

El desarrollo cognitivo correspondía al nivel intelectual normal en 15 niños (79\%), en tres a una inteligencia limítrofe y uno mostro retardo mental moderado. El rendimiento escolar fue muy bueno en dos niños, bueno en 11 , regular en dos y deficiente en cuatro pacientes.

De acuerdo a la clasificación multiaxial de Rutter, en la esfera psiquiátrica, tres niños se ubicaron en lo normal. En los 16 restantes (84\%) se detectaron reacciones de adaptación $(n=2)$; desórdenes emocionales - de orden depresivo 0 ansioso- $(n=5)$; desórdenes psicosomáticos $(n=3)$; trastomo de conducta $(n=1)$; trastornos en el desarrollo de la personalidad $(n=3)$; dislexia $(n=1)$, retardo mental $(n=1)$.

En la relación con los pares, seis niños no presentaba dificultades, 11 mostraban tendencia a ser inbibidos y dos se mostraban altamente competitivos.

En los aspectos afectivos (tablas 1 y 2), 14 de los 19 pacientes lograron cumplir la tarea en el cuento de animales y 18 niños en CAT-A. La percepción del mundo era amenazante para la mayoría de ellos. La percepción de sí mismo result 6 ser predominantemente negativa, sintiéndose desvalidos. En el cuento de animales, 
Tabla 1

Aspectos psicológicos en niños sobrevivientes de cáncer

\begin{tabular}{lcc}
\hline & $\begin{array}{c}\text { Cuentos de } \\
\text { animales }\end{array}$ & CAT-A \\
\hline $\begin{array}{l}\text { Número de niños } \\
\text { Percepción del mundo: }\end{array}$ & 14 & 18 \\
$\quad \begin{array}{l}\text { Amenazante } \\
\text { Amistosa }\end{array}$ & 11 & 15 \\
Percepción de sí mismo: & 3 & 3 \\
$\quad$ Predominante negativa & 11 & 13 \\
$\quad \begin{array}{l}\text { Positiva } \\
\text { Ansiedades principales: }\end{array}$ & 3 & 5 \\
$\quad$ Ansiedad de inuerte & 7 & 10 \\
$\quad$ Temor al daño físico & 3 & 2 \\
$\quad$ Temor a la desaprobación & 2 & 4 \\
$\quad$ Ansiedad de separación & 2 & 2 \\
\hline
\end{tabular}

Dimensión afectiva, evaluada a traves del cuento de aniInales en 14 riños y CAT-A. en [B.

Tabla 2

Aspectos psicológicos en niños sobrevivientes de cáncer

\begin{tabular}{|c|c|c|}
\hline & $\begin{array}{l}\text { Cuentos de } \\
\text { animales }\end{array}$ & CAT·A \\
\hline Número de nitĩos & 14 & 18 \\
\hline \multicolumn{3}{|l|}{ Tipo de vinculo: } \\
\hline Agresivo & 7 & I1 \\
\hline Amoroso y de protección & 6 & 5 \\
\hline Inestable & 0 & 1 \\
\hline Desvinculado & 1 & 1 \\
\hline \multicolumn{3}{|l|}{ Conflicto principal: } \\
\hline Temálica persecutoria & 8 & 11 \\
\hline De convivencia & 3 & 4 \\
\hline De extravío & 1 & - \\
\hline De comperencia & 1 & 3 \\
\hline No plantea & l & - \\
\hline \multicolumn{3}{|l|}{ Tipo de solución: } \\
\hline Positiva & 8 & 8 \\
\hline Negativa & 3 & 4 \\
\hline Sin solución & 3 & 6 \\
\hline
\end{tabular}

Dimensión afectiva, evaluada a través del cuento de animales en 14 niños y CAT-A, en 18.

tres se manifestaron agresivos, pero rechazados. Las ansiedades principales eran: en un número importante de los casos, la de muerte; en grado menor, el temor al daño físico, a la desaprobación y a la separación. En cinco niños (CAT-A) la ansiedad de muerte era muy intensa, desor- ganizándolos en su producción. En el tıpo de vínculo predominaba la temática persecutoria. Alrededor de la mitad de los niños resolvió el conflicto en forma positiva, una proporción de ellos dejó el final abierto (tres de 14 en el cuento de animales y seis de 18 en el CAT-A).

El contenido principal, en el total de los dibujos realizados por cada niño, fue en siete el lemor al daño físico o la recaída, en seis niños predominaba el sentimiento de soledad, mostrando además dificultades en el contacto con los pares, dos niños se centraban en los conflictos interpersonales al interior de sus familias, en otros dos niños predominaba la vivencia de armonia consigo mismos y en uno destacaban las situaciones de agresividad y desafío.

\section{Comentario}

Desde una perspectiva evolutiva, la muestra esiudiada se adscribe a la edad escolar, caracterizada en términos generales como un período en que se espera que el niño tenga vivencias de dominio del cuerpo $y$ vigor físico, a la par que sentimientos de seguridad al enfrentar y tener buen éxito en las tareas cognitivas y afectivas que le impone el sistema escolar ${ }^{10}$. En el área cognitiva, la distribución del nivel intelectual de la muestra, tomando en consideración su reducido tamaño, no difiere mayormente de la población general ${ }^{11}$. Existe correlación entre el resultado de las pruebas y el rendimiento escolar que, en la mayoría de los niños, fue satisfactorio.

Llama la atención la alı frecuencia de alteraciones psiquiátricas de diverso tipo, en comparación con una población normal ${ }^{12}$, lo que sugiere que sería recomendable hacer evaluaciones sistemáticas y proveer con el tratamiento pertinente a todos los niños afectados de cáncer, con el propósito de protegerles contra el establecimiento de trastornos de esta índole.

En investigaciones realizadas en Argentina, con el test CAT-A en escolares normales, con edades similares a los de nuestro grupo, se demostró que las historias contienen actividades cooperativas, sentimientos de independencia por parte del personaje infantil, así como desenlaces definidos, logrando la figura infantil escapar, a menudo, de los peligros ${ }^{13}$. Otros estudios con CAT-A en niños con problemas emocionales mostraron identificaciones con figuras que 
parecen inferiores, en historias con un medio agresivo y frustrante. El tema de la muerte aparece con frecuencia en niños ansiosos ${ }^{13}$. Con el cuento de animales, en las historias de los niños escolares normales predominan contenidos de solidaridad y percepción de sí mismos con fortaleza ${ }^{15}$. En los resultados obtenidos con el CAT-A y el cuento de animales en estos niños sobrevivientes de cáncer, no encontramos contradicciones importantes frente a las dimensiones definidas, tomando en consideración que el CAT-A agrega mayor riqueza de contenidos por la exposición de distintas situaciones, pero consideramos alta la frecuencia de los que muestran vivencias amenazantes del mundo que les rodea, percibiéndose al mismo tiempo desvalidos para enfrentar las dificultades. Estos resultados serian comparables, de acuerdo a la experiencia de los autores, con el comportamiento de niños con trastornos psiquiátricos de tipo ansioso y los interpretamos como consecuencias de la tendencia a retrotraerse que presentan algunos de estos niños, buscando refugio en sus padres.

Nos llama la atención que todos los niños con nefrectomia u orquidectomía se perciben negativamente a sí mismos, con una vivencia de fragilidad corporal. Muestran además mala relación con su grupo de pares. Estos resultados concuerdan con otras investigaciones que señalan a la secuela física como factor de riesgo para una positiva adaptación ${ }^{4}, 5,8$. Si bien la muestra es pequeña, estos resultados nos permiten plantear la necesidad de que estos niños puedan elaborar psicológicamente la pérdida de un 6rgano físico.

Por otra parte, consideramos que es alta la frccuencia de niños que presentan la ansiedad de muerte como ansiedad principal. En una muestra normal esta ansiedad aparece escasamente en el cuento de animales, asemejándose, sin embargo, a la patología psiquiátrica ansiosa. Pensamos que en el grupo estudiado la no resolución de los conflictos y la ansiedad de muerte pueden estar relacionadas con la persistencia del temor a la recaída, tanto en los niños como en sus familias, temática recurrente en los dibujos que realizaron.

La familia es la principal fuente de soporte psicológico en los niños pequeños, pero el deseo de olvidar y los propios temores sobre el futuro del hijo que tuvo cáncer, probablemente interfieran con la posibilidad de acoger las ansiedades de ellos. Concordamos con Oppenheim $^{8}$, quien plantea que la familia llena los vacíos de conocimiento acerca de la enfermedad con fantasías sobre el cáncer, traduciéndose en último término en mayores restricciones en la vida diaria de estos niños. Cabría esperar que así como durante el período de tratamiento la comunicación abierta entre el niño, su familia y e) equipo oncológico favorece el ajuste a la enfermedad ${ }^{5}$, el poder contrastar las dudas durante el período de remisión favorecerá la disminución de la ansiedad, incrementando de este modo la adaptación.

El método empleado, al dejar libre de expresión a la fantasía, permite pesquisar temores y ansiedades manifestadas siempre en la vida diaria y más difíciles de obtener a través de entrevistas. A la luz de estos resultados, en el tratamiento del niño con cáncer debe incluirse la evaluación psiquiátrica de rutina e intervenciones pertinentes para evitar trastornos secundarios posteriores. Además es preciso considerar que, tanto durante el tratamiento oncológico como en el seguimento a largo plazo, es indispensable mantener comunicación abierta entre el niño, su familia y el equipo multidisciplinario tratante, $\mathrm{el}$ que debe incluir psiquiatras y psicólogos.

\section{Resumen}

La mayoría de un grupo de niños menores de 11 años, sobrevivientes de cáncer infantil, percibía el mundo de un modo amenazante, predominando en ellos percepciones negativas de sí mismos. La ansiedad de muerte representó el principal tipo de ansiedad. Estos hallazgos nos permiten plantear la necesidad de considerar el correlato afectivo-vivencial durante la iniciación, tratamiento y controles, en lodos los niños con enfermedad oncológica, prestando apoyo e información a ellos y a sus familiares.

(Palabras clave: cáncer, neoplasmas, niños, sobrevivencia, aspectos emocionales, afectivos, calidad de vida.)

\section{Agradecimientos}

A Ia Drá. Minarn Campbell, cuya preocupacióa por el control alejado de los nínos mejorados de cáncer hizo posible este estudio. 


\section{Referencias}

I. Birch JM. Marsden HB, Morris Jones PH. Pearson D, Blair V: Improvements in Survival from Childhood Cancer: Results of a population based survey over 30 years. Br Med J 1988: 296: 1372-1,376.

2. Mulhem $R$. Worsermun A, Friednum A, Fairclough $A$ : Social Competence and Behavioral Adjustment of Children who are longterm Survivors of Cancer. Pediatrics 1989: 83: 18.25.

3. Ullet F: Aspectos psicosociales del cáncer en tuños. Rev Chil Pediatr 1993; 64: 5.5-62

4. Wasternuan AL. Thompson E. Wilinas I. Fairclough D: The Psychosocial status of survivors of childtood Adolcscent Hodgkun's Disease. Am J Dis Child 1987; 141: $626 \cdot 631$

5. Glacer JP: Psychiatric Aspects of Cancer in Childhood and Adolesence. En: Lewis M.. Child and Adolescent Psychiatry. Baltimore: Williams and Wilkins, 1991: 964-977.

6. Roma $E$, Varyas L: Problemas psicologicos en la familia del niño con cáncer. Rev Chil Pediatr 1992: 63: 222-229.

7. Brown R. Kastow NJ. Hazzard AP, et al.: Psychiatric and Family Functioning in Children with lukemia and their Parents. Am Acad Child Adolescent Psychiatry 1992: 31: 495-502.

8. Oppenhein D: Approche des Sequelles psychologiques des enfants cancercux et de leurs familles. Neuropsychiatric de l'Enfance 1989; 37: 347-351,

9. Lansky Sh. Cairs N N. Hassanein $R$, Wehr $J$, Lomman $J$ : Childhood Cancer: Parenteral discord and divorce. Pediatrics 1978; 62: 184-188

10. Lidz Th: La persona, su desarrollo a través del ciclo vital. Barcelona: Editorial Herder, 1985; 324-362.

11. Wechster D: Tesı de Inteligencia para niños (WISC). Bs. Ajres: Editorial Paidos, 1983; 27-28.

12. Ullow $F$. Funes $F$. Montenegro P: Prevalencia de desórdenes psiquiátricos en Pobjación de 5 a 15 anos que ingresa al Servicio de Cirugía del Hospitat Robetto del Río. Revista de Psiquiatría Chile 1992; 3-4: 12041212 .

13. Buringoltz S. Frank $R$, Menéndez F: Estudios nonnativos. En: El CAT en el psicodiagnóstico de niños. Bs. Aircs: Ediciones Nueva Visión, 1984; $31-49$.

14. Figuri $P$. Ievir $D$ : Descripción de cuentos de animales sreados por niños escolares nomales. Memoria para optar a tírulo de Psicólogo, Universidad Gabtiela Misiral. 1993.

\section{AVISO A LOS AUTORES}

Por acuerdo del Comité Editorial, la Revista Chílena de Pediatría devolverá sin tramitar todos los trabajos que no den estricto cumplimiento al Reglamento de Publicaciones y a las Instrucciones de los Autores que se editan en cada número de la Revista. 